\title{
La violencia en el noviazgo, los indicios que no se visibilizan.
}

\author{
Violence in courtship, the signs that are not visible.
}

\section{María Edith Gómez-Gamero a, Julissa Rodríguez-Hernández ${ }^{b}$, Aylin Quintanar-Vázquez ${ }^{c}$}

\begin{abstract}
:
Violence in courtship is a phenomenon that progresses silently and that the actions, acts or forms of violence that occur in a relationship are not visible, in this study made to university students, it is elucidated from the perspective of violence against women. relationship that studies the origins of violence in couples with a relationship of courtship, in general unmistakable acts are identified, which comes from a more subtle violence in this relationship. The efforts of the specialists oriented towards the prevention of dating violence are oriented, in part, towards the detection of the first signs of violence that may manifest themselves at the beginning of it. Many teenagers create great expectations around courtship, such as love, care, support and understanding; so that young people who live violent relationships are difficult to realize this situation, and can reach a point where the courtship becomes a torment or even more complex situations, to death. Violence in courtship is a serious situation that does not respect social strata, cultures, or age ranges. Physical violence can occur with blows, pinching, pushing, among other things; verbal violence in insults, threats, offensive words, there are different manifestations, the objective is to identify this phenomenon in time.
\end{abstract}

\section{Keywords:}

Violence, courtship, couple

\begin{abstract}
Resumen:
La violencia en el noviazgo es un fenómeno que avanza silenciosamente y que no se visibilizan las acciones, actos o formas de violencia que se producen en una relación, en este estudio realizado a estudiantes universitarios se dilucida desde la visión de la violencia contra la mujer esta relación que estudia los orígenes de la violencia en parejas con una relación de noviazgo, en general se identifican actos inconfundibles, que proviene de una violencia más sutil en esta relación. Los esfuerzos de los especialistas orientados hacia la prevención de la violencia en el noviazgo se orientan, en parte, hacia la detección de las primeras señales de violencia que pueden manifestarse al inicio de la misma. Muchos adolescentes crean grandes expectativas alrededor del noviazgo, como lo son el amor, el cuidado, el apoyo y la compresión; por lo que a los jóvenes que viven relaciones violentas se les dificulta darse cuenta de dicha situación, y puede llegar a un punto en el que el noviazgo se convierta en un tormento o incluso a situaciones más complejas, a la muerte. La violencia en el noviazgo es una situación de carácter serio, que no respeta estratos sociales, culturas, ni rangos de edades. La violencia física puede presentarse con golpes, pellizcos, empujones, entre otras cosas; la violencia verbal en insultos, amenazas, palabras ofensivas, existen diferentes manifestaciones el objetivo es identificar este fenómeno a tiempo.
\end{abstract}

\section{Palabras Clave:}

Violencia, noviazgo, pareja

\section{Introducción}

La violencia en el noviazgo es un fenómeno que suele producirse en edades tempranas, el noviazgo tiene su inicio en la adolescencia, edad crítica que va unida a la búsqueda de una identidad social, profesional, sexual y de género. Es esta etapa se asientan los estereotipos de género y, por tanto la masculinidad tradicional, así como

\footnotetext{
a Autor de Correspondencia, Universidad Autónoma del Estado de Hidalgo, Escuela Superior de Actopan, https://orcid.org/0000,0002-76999995, Email: maria_gomez6500@uaeh.edu.mx

b Universidad Autónoma del Estado de Hidalgo, Escuela Superior de Actopan, Email: aileeeen26@gmail.com

c Universidad Autónoma del Estado de Hidalgo, Escuela Superior de Actopan, Email: yulissiitha13@gmail.com
} 
los mitos sobre el amor, la amistad, la pareja y la familia. La adolescencia forma una parte importante de esta etapa (noviazgo), es un período durante el cual las personas mantienen una relación de estado amoroso, en el que se vive una gran cantidad de experiencias y en donde también se sufre de violencia, según el (CONAVIM, 2018) este es un fenómeno en el que se hace uso de la fuerza para conseguir un fin, especialmente para dominar a alguien o imponer algo, o bien imponer para obtener algo por la fuerza, desafortunadamente puede ser debido a la ignorancia de muchos de los jóvenes, ya que en su mayoría se permite esta actitud de la pareja en el noviazgo y se trata de justificar, sin considerar que es un tipo de violencia.

La violencia se presenta de varias formas como son: física, psicológica, sexual, verbal, simbólica, económica, entre otras. Este fenómeno está inmerso en la gran mayoría de las relaciones de noviazgo en adolescentes, al iniciar una relación de pareja que provoca una variedad de problemas principalmente sentimentales, comúnmente el sexo femenino se encuentra inmerso en situaciones de vulnerabilidad, como consecuencia de esta relación de pareja las mujeres pueden presentar baja autoestima, asociada a otras consecuencias por el daño físico producido por golpes y maltratos y problemas para socializar por los daños psicológicos resultado de esta relación que incluso puede dañar la vida de las mujeres.

Los efectos de la violencia suelen ser el algunos casos depresión, problemas familiares y el hecho de que alguno de los dos integrantes de la pareja o los dos consuman alcohol o alguna otra sustancia misma que genere agresión física, verbal o psicológica en la pareja y generalmente esta condición se utilice para justificar y decir "no era yo" antes de aceptar el error y reconocer que es violencia, los actos inapropiados conllevan a actos violentos que pueden acrecentarse y generar más violencia. La violencia de pareja se ha posicionado como uno de los problemas que enfrenta la sociedad y como un problema de salud en el mundo, no sólo por la enorme magnitud del fenómeno sino también por la gravedad de las consecuencias personales y sociales que esto trae aparejado. Algunas mujeres maltratadas al respecto señalan que el "descubrir" la violencia en sus relaciones es remontarse a la etapa del noviazgo en donde están presentes importantes factores e indicios de la violencia que no se visibilizan a tiempo.

En la sociedad mexicana, existe la creencia sobre todo aceptada que el noviazgo es la época "ideal" de una pareja, donde se vive cada momento con intensidad, alegría y pasión; también, se acepta como natural que con la convivencia, las cosas cambien y que poco a poco de aquella época romántica sólo queden recuerdos (Ligeia,
2011). Sin embargo, la realidad se presenta diferente, porque es durante ésta etapa en donde las jóvenes parejas se enfrentan a situaciones violentas que poco tienen que ver con lo que se espera de una relación en la que se tienen expectativas muy diferentes a lo que en realidad se vive. El problema de la violencia en el noviazgo ha sido poco estudiado, Pero se han realizado estudios sobre el actual reconocimiento de que ciertas actitudes y conductas agresivas en el noviazgo constituye un problema social y de salud, que de ninguna manera implica que sólo haya existido de manera reciente, sino simplemente hasta hace poco el tema ha sido objeto de investigación sistemática y de intervención, en tiempos pasados el fenómeno era algo que se dejaba pasar por alto puesto que en la mayoría de los casos esta se presenta del hombre hacia la mujer, justificando que la mujer hace algo mal y el hombre por encontrarse en una posición de poder aceptado socialmente se toma el derecho de que su pareja debe hacer lo que él diga y mande, sin considerar que estas actitudes forman parte de lo que ya se establece en la legislación como un tipo de violencia, actualmente existen una gran cantidad de legislaciones vigentes al respecto a nivel Nacional e Internacional que promueven la no violencia y se legista al respecto con el fin de erradicar estas prácticas y en las que se apoya a la mujer, esto revisten de mayor relevancia ante este fenómeno pero a pesar de estas nuevas condiciones que protegen a las mujeres, la cultura influencia estas prácticas y predomina en las sociedades patriarcales en donde se concibe a la mujer como la acreedora del género denominado "débil" en el que se fortalece el machismo y la aceptación tácita de estas prácticas y la forma de dominación del hombre sobre la mujer, incluso de las propias mujeres en las que se acepta la violencia como una práctica normal, esto implica que tanto entre los hombres como entre las mujeres la violencia se entrevea como un fenómeno normal, común y con los jóvenes esta acción se presente como producto de lo que se ha aprendido a lo largo de la vida.

Desde la perspectiva feminista existe mayor probabilidad de que en una relación de pareja en donde existan actos violentos la mujer sea la que se encuentre en un estado más vulnerable, es probable que en la mayoría de las relaciones de noviazgo exista el fenómeno de la violencia, y este presente en las personas provienen de una familia en la que comúnmente se ha vivido algún tipo de violencia en el seno familiar y este patrón se está repitiendo.

En el noviazgo o previo a él, la etapa del enamoramiento es en la que pueden presentarse signos o indicios de la violencia ya que es más permisiva esta práctica en la que se pueden presentar algunas conductas que conllevan la violencia implícita pero que son ignoradas por completo, 
que incluso puedan callarse y aceptarse tácitamente sin hacer pública esta condición, incluso con los propios familiares debido a la no existencia de una comunicación que permita que estas situaciones puedan prevenir o detenerse porque la misma familia considera que esto pasa en todas las relaciones de noviazgo y que la mujer tiene la obligación de soportar estas condiciones normalizándose la violencia formando parte de las prácticas socioculturales de una sociedad. Cuando los indicios de violencia se ocultan a familiares, se fortalece la violencia ya que no se da a conocer fuera de la relación o al menos no a alguna persona que pueda intervenir y apoyar para poner un alto a la misma solo se asume como parte de la relación.

Según la (OMS, 2002) el fenómeno de la violencia es ya una problemática que va creciendo día a día, cada año, más de 1.6 millones de personas en todo el mundo pierde la vida violentamente y es una de las principales causas de muerte en poblaciones entre los 15 y 44 años así como la responsable del $14 \%$ de las defunciones en la población masculina y el $7 \%$ de la femenina, por lo tanto es necesario visibilizarla para que las mujeres identifiquen en que momento están sufriendo de violencia y terminar o poner un alto a una relación que puede traer consigo mayores problemas conforme esta avanza. Sin embargo la violencia hombre-victimario de la misma manera se ha incrementado y por lo tanto es necesario estar informados sobre lo que se tipifica como violencia para evitar este tipo de conductas que dañan física y psicológicamente a las personas, que por el contrario en una relación sana se fortalecen los lazos de comunicación y confianza entre las parejas.

\section{Identificando la violencia en el noviazgo}

¿En dónde estuviste?, ¿Por qué no me contestas el teléfono? son algunas preguntas en la pareja que van indicando que se está viviendo una relación de violencia aunque estas sean preguntas aparentemente inofensivas y se realicen justificando desde el discurso "para saber en dónde estás y pueda protegerte". La violencia durante el noviazgo esta incrementándose en nuestro país, las jóvenes aceptan ser víctimas de violencia, y reconocen que las agresiones y actos violentos pasaron desapercibidos en los momentos en que ocurrieron. Ante el crecimiento de este tipo de actos violentos es necesario que se aprenda a reconocer y diferenciar una actitud o comportamiento violento o agresivo de parte de la pareja. Así mismo es de vital importancia que los adolescentes reconozcan que son valiosos e importantes, que son individuos que requieren de respeto, cariño y comprensión, que no necesitan estar al lado de una persona para sentirse plenos y felices. El concientizar a los jóvenes sobre las conductas violentas e identificación de los indicios en una relación de noviazgo coadyuva a estar en condiciones de diferenciar entre el amor y lo que es sometimiento y dominio, es reconocer los signos de violencia para detenerla oportunamente, para construir una cultura basada en el respeto, la tolerancia y la equidad de género entre hombres y mujeres, las legislaciones que se han generado a partir de estas condiciones forman parte de implementar una cultura en contra de la violencia. El proponer alternativas que generen en el noviazgo actitudes propositivas, que favorezcan la convivencia con respeto a los derechos de la otra persona, a la diferencia, a la tolerancia, fomentando la capacidad de diálogo, la escucha profunda, donde la opinión de la otra persona sea valorada; es fundamental para una relación sana de noviazgo.

La violencia en el noviazgo es fenómeno común en nuestra sociedad que no es ajeno al contexto escolar, en los pasillos o en las aulas y fuera de ellas se hace uso del lenguaje obsceno entre parejas, se pueden observar humillaciones e incluso empujones, por lo que es imprescindible establecer acciones que permitan identificar los síntomas de estar inmersos en una relación no basada en el respeto para hacer frente a este tipo de situaciones. La violencia contra las mujeres debe visibilizarse debido a que la propia cultura permite que esta se reproduzca, sin embargo identificar los signos de violencia contra la mujer permite que las mujeres además de identificarla estén en condiciones de poner un alto e irse empoderando para afrontar con más recursos personales el fenómeno de la violencia.

El noviazgo de acuerdo al (CONAVIM, 2018) es un período en el que dos personas comparten una relación íntima, esta puede ser sexual, pero no necesariamente tiene que serlo, puede ser casual, seria, heterosexual o entre personas del mismo sexo, con compromiso, sin él, de corta o larga duración, se caracteriza por ser un período de entendimiento de una pareja, en la que se desean cubrir expectativas con respecto a la formación de algo más sólido y estable para finalmente fundar una familia, el noviazgo puede iniciar en la adolescencia con una amistad sentimental, que lleva un proceso, y es un ciclo: en el que se presenta primero la amistad, luego el noviazgo y después se da el rompimiento de las relaciones, y con cada rompimiento puede reiniciar una nueva relación, un nuevo noviazgo a excepción de la relación con persona con quién se decide casar.

En el noviazgo se puede presentar también el amor sexual, lo que se suele denominar también amor romántico o pasional, tanto por su conceptualización como por la educación y experiencias amorosas que promueve, pero que es parte intrínseca de la subordinación social de las mujeres. 
La pasión amorosa es definida por Jankowiak (1995) se define en base a cuatro elementos: idealización, erotización del otro, deseo de intimidad y expectativa de futuro, en una formulación pretendidamente universal que tomamos como nuestra, no tanto porque dichos elementos no estén o no puedan estar presentes en otras formas de amor (materno/paterno, filial, amistad...), sino por el tratamiento específico que tienen dentro de la sociedad occidental cuando se refieren al amor sexual.

Para Platón el amor se concebía como el profundo conocimiento de la otra persona después de la visión. Existen diferentes palabras en la actualidad que definen una relación de pareja o noviazgo:

- Salir

- Salir juntos

- Estar con alguien

- Estarse viendo con alguien

- Solo amigos

- Amigos con beneficios

- Involucrados

- Enrollarse

- Tener un compromiso

- Tener una relación

Sin embargo la violencia en el noviazgo ocurre en una relación amorosa cuando uno de los integrantes de la pareja abusa física, emocional, psicológica o sexualmente para dominar y mantener el control sobre la otra y el abuso se produce por el hecho de estar enamorados justificando que en ocasiones esto hace perder la propia identidad y ejercer violencia o ser víctima de esta. Algunos autores señalan que las relaciones de noviazgo en donde la subjetividad está muy presente, para Hernando (2000) es "el modo en que las mujeres internalizamos, actuamos y reproducimos un conjunto de valores y actitudes a través de las cuales nosotras mismas contribuimos a perpetuar esa situación de desigualdad respecto a los hombres", Para (Ligeia, 2011) el maltrato a la pareja puede ocurrir en cualquier momento, desde la primera salida juntos 0 puede presentarse incluso transcurridos varios años de relación, pero la diferencia ante otros tipos de violencia es precisamente en el momento que se hace presente y la forma en la que esta se hace visible incluso no ante la pareja sino ante los demás que se encuentran en su entorno, la violencia puede ejercerse en el proceso de socialización que en ocasiones lleva implícita la dominación debido a el hecho socialmente aceptado de ser mujer y el rol o estereotipo de género la refuerzan, mismos que determinan el dominio como comportamiento aceptado del género masculino y la sumisión del femenino, sumados a la idealización del "amor romántico" que todo lo puede superar y todo lo perdona, así como por el carácter informal y efímero de la relación.
Aunque tales patrones de conducta sean parecidos a los identificados en parejas formales, no es tan frecuente que en el noviazgo se presenten niveles de maltrato físico similares a los del matrimonio o relaciones equivalentes; sin embargo no se está exento de que puede suscitarse es decir, entre novios los golpes no son la manifestación ordinaria de la violencia, pero en el ámbito del "juego" son comunes, y eso lleva a que su gravedad parezca mínima. Las conductas violentas en las relaciones de pareja no formales no son percibidas como tales ni por las víctimas ni por los agresores, pues generalmente se confunden maltrato y ofensas con amor e interés por la pareja. Así la violencia adopta diferentes expresiones desde la ofensa verbal hasta el homicidio y puede adoptar múltiples rostros: insultos, gritos, amenazas, empujones, puntapiés, intimidaciones, castigos, sometimiento, bofetadas, puñetazos, hasta las agresiones con armas. Según (Ligeia, 2011) varias encuestas indican que nueve de cada diez mujeres en la actualidad, viven violencia en sus relaciones amorosas, pues por "amor", que ellas toleran el maltrato confiadas en que algún día su pareja cambiará, sin embargo esta condición no logra erradicarse y la violencia genera más violencia, en el ámbito más íntimo, en el sexual, el tipo más frecuente de esta agresión es la llamada violación por confianza, que sucede cuando las jóvenes o adolescentes son presionadas a tener actividad sexual generalmente por la pareja hombre y en la mayoría de casos con parte del consentimiento de ellas; esto se ejemplifica con la famosa "prueba del amor", que constituye una de las principales formas de violencia que los jóvenes ejercen sobre sus "novias", quiénes finalmente aceptan sostener relaciones sexuales más por presión que por convencimiento propio o aún en contra de sus deseos.

Los tipos de violencia ya se encuentran tipificados con la finalidad de erradicar estas conductas en las relaciones de pareja. La violencia en las relaciones de pareja está ligada a la renuncia de la libertad y las aspiraciones, se generan a partir de la continuidad de los roles como son la maternidad, la familia en donde el amor romántico otorga a la mujer la libertad de elegir pareja, pero la libertad se queda corta o atrapada en los roles de esposa o madre, la emancipación femenina no se logra porque el amor romántico se relaciona directamente con los roles o estereotipos de género, la belleza, el amor único, ser madre, la familia, la virginidad que la frustran para alcanzar la felicidad prometida y por lo tanto el amor puede convertirse en insatisfacción. Las relaciones de pareja tienen un punto de inicio en donde se establecen las formas de negociación del poder, que pueden o no predisponer que se manifieste la violencia al momento de integrarse ya como familia o una pareja que está en la 
etapa preparatoria del proceso que se reconoce como el noviazgo.

El 27 de marzo del 2009 se estableció para los 31 Estados en la República Mexicana la Ley de Acceso a las mujeres para una vida libre de violencia (SEGOB, 2007) en los que se tipifican los tipos de violencia categorizándolas en:

Violencia familiar: Es el acto abusivo de poder u omisión intencional, dirigido a dominar, someter, controlar, o agredir de manera física, verbal, psicológica, patrimonial, económica y sexual a las mujeres, dentro o fuera del domicilio familiar, cuyo agresor tenga 0 haya tenido relación de parentesco por consanguinidad 0 afinidad, de matrimonio, concubinato 0 mantengan o hayan mantenido una relación de hecho.

Violencia laboral: Es la negativa ilegal a contratar a la víctima o a respetar su permanencia o condiciones generales de trabajo; la descalificación del trabajo realizado, las amenazas, la intimidación, las humillaciones, la explotación y todo tipo de discriminación por condición de género.

Violencia docente: Son aquellas conductas que dañen la autoestima de las alumnas con actos de discriminación por su sexo, edad, condición social, académica, limitaciones y/o características físicas que les infligen maestras o maestros.

Hostigamiento sexual: Es el ejercicio del poder, en una relación de subordinación real de la víctima frente al agresor en los ámbitos laboral y/o escolar. Se expresa en conductas verbales, físicas o ambas, relacionadas con la sexualidad de connotación lasciva.

Acoso sexual: Es una forma de violencia en la que, si bien no existe la subordinación, hay un ejercicio abusivo de poder que conlleva a un estado de indefensión y de riesgo para la víctima, independientemente de que se realice en uno o varios eventos.

Violencia en la comunidad: Son los actos individuales 0 colectivos que transgreden derechos fundamentales de las mujeres y propician su denigración, discriminación o exclusión en el ámbito público.

Violencia institucional: Son los actos u omisiones de las y los servidores públicos de cualquier orden de gobierno que discriminen o tengan como fin dilatar, obstaculizar o impedir el goce y ejercicio de los derechos humanos de las mujeres [...] así como su acceso al disfrute de políticas públicas destinadas a prevenir, atender, investigar, sancionar y erradicar los diferentes tipos de violencia.

8.Violencia feminicida: Es la forma extrema de violencia de género contra las mujeres, producto de la violación de sus derechos humanos, en los ámbitos público y privado, conformada por el conjunto de conductas misóginas que pueden conllevar impunidad social y del Estado y puede culminar en homicidio y otras formas de muerte violenta de mujeres.

Para (Ligeia, 2011) desde la niñez y en el seno familiar se aprende el juego de la negociación del poder en la pareja, en el seno familiar se produce y reproducen los roles y estereotipos, los niños aprenden a reconocer sus aspiraciones asignadas culturalmente a su sexo, se reproducen los roles dominante y dominado, los jóvenes crecen aprendiendo a amar de manera confusa en donde el conflicto, la manipulación, el autoritarismo, incluso el temor forma parte de este aprendizaje, en donde padre y madre los aman y "por eso les hacen daño" con los castigos, presentándose la violencia como algo cotidiano en donde para resolver un conflicto y reclamar espacios está ligada a la visión de que el hombre sustenta la autoridad y es normal ejercer la violencia, donde el autoritarismo es una alternativa para resolver los conflictos entre la personas y se aprende a vivir entre la violencia y así se normaliza. La violencia se asimila socialmente y es aprendida, el hombre aprendió a ejercerla y la mujer a aceptarla y se transmite socialmente a través de las diferentes instituciones de la sociedad. Las mujeres en tanto se conciben como un grupo desfavorecido por razones derivadas del orden de género dominante (CONAVIM, 2018). En la familia el papel fundamental se centra en el desarrollo de la persona y es dónde se conforma la identidad, la femenina se transmite en la relación abuela-nieta, madre-hija, sobrina-tia, hermana-hermana, amiga-amiga y la identidad masculina se estructura de la misma manera relacionándose con sus iguales. (Ligeia, 2011)

Los jóvenes en la relación de noviazgo repiten los roles tradicionales, los roles de violencia que son relaciones afectivas que han germinado en un entorno de violencia en el que se ha aprendido a ser violento. Es así como la violencia se conforma como el hilo conductor para su relación y futuras relaciones como el matrimonio y la familia. Es así como la relación entre hombre y mujer queda marcada por una relación de inequidad y desigualdad que se legitima por la autoridad fincada en el rol masculino de tal forma que no es perceptible simplemente es asumida y ejercida en la relación como parte de la misma.

Una persona violenta seguirá reproduciendo esta conducta por estar aprendida e introyectada en su personalidad y la pareja no debe esperar a que esto cambie de ahí la importancia de reconocerla. En las parejas se presenta también el ciclo de la violencia y se asume como algo normal concibiéndose erróneamente como parte de la misma relación, normaliza y para hacer 
un alto a la misma es necesario identificarla y entender lo que está pasando, conocer que se está viviendo en una relación violenta y sobre todo es importante tomar la decisión de romper con este ciclo violento, alejarse, pedir ayuda a un profesional especializado y en su caso también hacer uso de la denuncia.

Las conductas que son violentas para nada enriquecen ni estimulan la relación, al contrario la enferman y dañan emocionalmente, psicológicamente y físicamente llegando incluso a la muerte.

El círculo de la violencia se presenta en las siguientes fases:

Fase I: De acumulación de tensión. Se presentan pequeños episodios que llevan a roces permanentes entre los miembros.

Fase II: Del golpe. En esta etapa la mujer, frente al golpe, puede llevar a cabo la denuncia judicial.

Fase III: De idealización o luna de miel. En esta etapa se produce el arrepentimiento por parte de él, la mujer lo perdona y vuelve a creer en su pareja.

Estas fases se señalan de acuerdo al (CONAVIM, 2018), con la finalidad de identificarlas ya que se caracterizan por concebir que la vida en pareja todo es amor y color de rosa, pero en algún momento la mujer imagina que hizo algo que ha provocado la violencia del otro, se culpabiliza y asume que no debe hacer enojar a su pareja y es cuando se refuerza la etapa violenta que en algunos casos puede llegar a la muerte después de un ataque violento, se pasa a la siguiente etapa que es del arrepentimiento en la que generalmente se solicita de parte del hombre el perdón a la pareja y se promete jamás volver a golpearla pero esto solo es parte de un círculo vicioso del que muchas mujeres no salen, porque perdonan al agresor y consideran que esto no va a volver a suceder porque se lo ha prometido, sin embargo este ciclo de la violencia tiene más probabilidades de repetirse que de erradicarse, mientras se siga en esa relación violenta.

A continuación se muestra el gráfico del ciclo de la violencia:

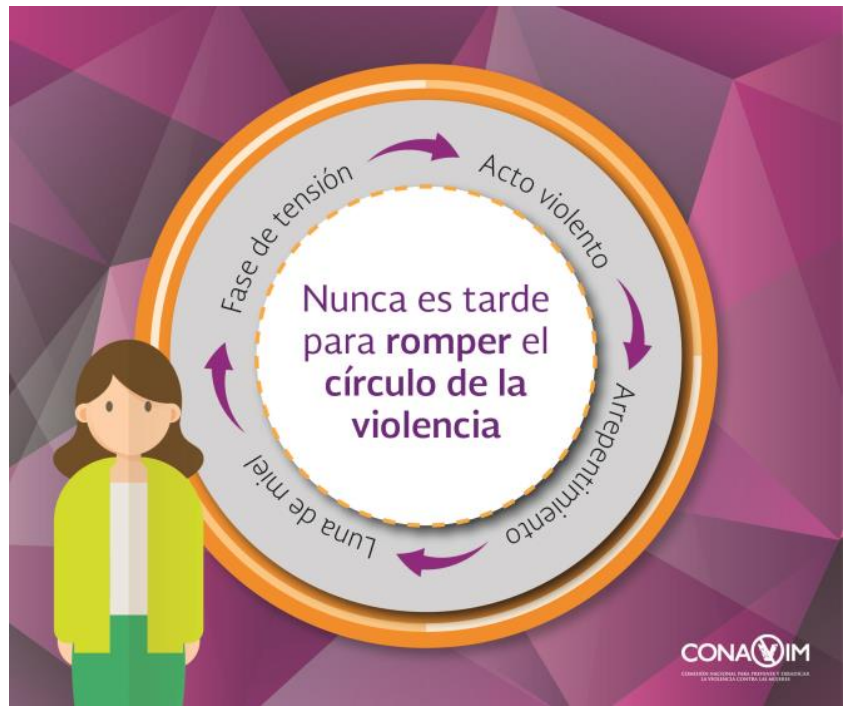

Figure 1. Ciclo de la violencia en el noviazgo FUENTE(CONAVIM,2018)

La violencia física, como emocional es la que más comúnmente se presenta en las relaciones de noviazgo, una forma común de ejercerla es mediante los celos que es un elemento matizado entre bromas, jugueteos y en donde la broma es un elemento para controlar, criticar y limitar a la pareja, esta es tolerada bajo este nuevo rol que lleva en el fondo la estructura del poder patriarcal de la dominación masculina, los jóvenes la aceptan porque es la etapa preparatoria para el matrimonio y en donde aprenden a conocerse y esperan cubrir con los roles de padre y madre, la violencia puede pasar desapercibida, puede esconderse detrás de la máscara del amor más cortés, más romántico y mantenerse oculta en la aceptación resignada. La violencia ante esta conductas se fortalece sobre todo al mantenerse oculta, tolerada, interiorizada, se reproduce en las prácticas y convencionalismos o discursos sociales, y se mantiene ahí agazapada bajo el sutil disfraz del inocente jugueteo amoroso (Ligeia, 2011). El noviazgo es en sí un escenario en el que se puede observar y conocer la forma en que las relaciones de género son afectadas por la violencia entre el juego como una forma de relacionarse entre los sexos en los que sigue permeando la violencia. El maltrato suele comenzar con el abuso psicológico difícil de identificar por estar enmascarada de cariño y afecto pero los comportamientos restrictivos o controladores socavan la capacidad de decisión y autonomía de las mujeres (se censura su forma de vestir, amistades, horarios, entre otros) estas conductas con el paso del tiempo producen dependencia y aislamiento, no se perciben como agresivas, se interpretan como prueba de amor pero desarrollan una situación de vulnerabilidad y dominación del hombre hacia la mujer. Existen algunos indicios de que una relación en el noviazgo es violenta, pero no se 
concibe como tal, algunos de estos se exigen en ciertas conductas que son indicios de la violencia como las siguientes:

- Controla todo lo que haces y pide explicaciones detalladas

- Cela e insinúa que andas con alguien más

- Revisa tus pertenencias: tu diario, celular, correo electrónico, mensajes

- Vigila y critica tu manera de vestir

- Realiza comparaciones tuyas con sus ex

- Desvaloriza, ofende y descalifica

- No responde si se le exige una explicación

- Siempre culpa a los demás de estar en contra de la relación

- Se niega a conversar acerca de los conflictos o desacuerdos de la pareja

- Obliga a hacer cosas que no quieres

- Amenaza con dejar la relación cuando no haces lo que quiere

- Coquetea con otras personas delante de ti o en secreto

- Presiona para tener relaciones sexuales.

Estos son solo algunos de los indicios en los que se involucra la violencia pero no se visibiliza como tal, pero que muestran que se está viviendo en una relación de violencia, es importante hacer una alto y no permitir que esta se siga reproduciendo y permitiendo.

Atendiendo a las legislaciones internacionales entre ellos a la Carta a los Derechos Humanos en donde se acentúa la igualdad de los derechos de la mujer en su Artículo primero se señala como uno de los objetivos de la ONU (Organización de las Naciones Unidas) buscar la Cooperación Internacional en el desarrollo y estímulo del respeto a los derechos humanos y a las libertades fundamentales de todas las personas.

Desde 1979 en la Convención sobre la Eliminación de todas las formas de Discriminación contra la mujer, se establecieron comités que supervisan el cumplimiento de estos acuerdos y distintos tratados por parte de los Estados miembros para la eliminación de la discriminación y violencia en contra de la mujer.

En 1994 en la Convención de Belém do Pará suscitada por la OEA (Organización de Estados Americanos) señala en su Artículo 10. Que violencia contra la mujer es cualquier acción o conducta, basada en su género que cause la muerte, daño o sufrimiento físico, sexual o psicológico en la mujer, tanto en el ámbito público como en el privado. El objetivo fundamental se encuentra en combatir la discriminación de la mujer en todas las esferas del desarrollo, todas las acciones encaminadas a un fin mundial en donde todos las países se comprometan a que las mujeres puedan acceder a una igualdad con los hombres y con ello ejercer sus derechos humanos garantizados.

Las Naciones Unidas han organizado cuatro conferencias mundiales sobre la mujer, que se celebraron en la Ciudad de México (1975), Copenhague (1980), Nairobi (1985) y Beijing (1995). A ésta última siguió una serie de exámenes quinquenales. En Beijing, China en la cuarta Conferencia Mundial sobre la Mujer, se estableció el compromiso por defender los derechos y la dignidad humana intrínseca de las mujeres y los hombres y la eliminación de todas las formas de discriminación de la mujer, y la declaración sobre la eliminación de la violencia contra la mujer y el derecho a su desarrollo.

Estos instrumentos de los derechos humanos rompen con el sistema de jerarquías, subordinación y discriminación entre los géneros comprometiendo a los Estados y haciendo extensivo a la sociedad el respeto a las normas, costumbres y prácticas que garanticen una real igualdad entre el hombre y la mujer.

En México como miembro de la ONU se han adoptado los acuerdos y declaraciones emitidas al respecto por estas organizaciones emprendiendo las acciones encaminadas a fortalecer los derechos humanos y discriminación.

\section{Conclusiones}

La violencia contra la mujer pretende erradicarse con acciones emprendidas desde organismos internacionales que legislan en contra de estas prácticas que vulneran los derechos de la mujer y que se llevan a cabo comúnmente en la vida cotidiana sin que se visibilicen y tampoco se ubique que se está viviendo una situación de violencia, es importante evitar cualquier interacción que contenga relaciones asimétricas, mediadas por el género y el poder e identificar en el noviazgo etapa encaminada a establecer lazos más fuertes para fundar finalmente una familia, las relaciones de violencia y a pesar de estar mediada por el contexto histórico y social por la dominación masculina y afirmada en las estructuras sociales, una relación de desigualdad e iniquidad puede establecerse desde el inicio de una relación con nuevas formas de convivencia más sanas, más modernas y nuevos papeles para cada uno con el fin de encaminar un noviazgo en el que imperen las relaciones de igualdad y no de dominación y sujeción, con un tono más permisible sobre acuerdos que lleven a establecer una familia fundada en vínculos más fuertes alejados de la violencia. 


\section{References}

[1] Amorós, P. C. (1988). Mujeres, feminismo y poder. Madrid: Forum de Política Feminista

[2] Bordieu, Pierre, La Dominación Masculina, Editorial Anagrama, Barcelona 2000

[3] Burin, M. (2007) El techo de cristal. Perspectivas psicoanalíticas sobre las mujeres y el poder "El techo de cristal en la carrera laboral de las mujeres. Acerca del deseo de poder en las mujeres". Buenos Aires, Lumen.

[4] Conavim (2018) Estudio representaciones y violencia vs mujeres en mediosdigitaleshttps://www.gob.mx/cms/uploads/attachment/file/3146 94/Estudiorepresentacione_y_violencia_vs_mujeres_en_medios_digita les.pdf

[5] Duque Elena, Aprendiendo para el Amor o para la violencia: Las relaciones en las discotecas., Editorial El Roure, Barcelona, 2006

[6] Giddens Anthony, La trasformación de la intimidad: Sexualidad, amor, erotismos en las sociedades modernas, Editorial Cátedra, Madrid, 1995.

[7] Lagarde (2015), R. M. (2015). Los cautiverios de las mujeres: Madre esposas, monjas, putas, presas y locas.

[8] Rubin, G. (1986). El tráfico de mujeres: Notas sobre la economía política del sexo. México.

[9] Mainwaring, S., Scully, T., \& Corporación de Investigaciones Económicas para Latinoamérica. (1996). La construcción de instituciones democráticas: Sistemas de partidos en América Latina. Santiago, Chile: CIEPLAN.

[10] Valcárcel, A. (2014). Feminismo en el mundo global. Madrid: Difusora Larousse - Ediciones Cátedra.

[11] In Valcárcel, A., In Renau, D., \& In Romero, R. (2000). Los Desafíos del feminismo ante el siglo XXI. Sevilla: Instituto Andaluz de la Mujer.

[12] Pascual F, A. (2016).Revista de Educación e Humanidades. Sobre el mito del amor romántico. Amores cinematográficos

y educación, (10), 63-78. Recuperado de https://www.google.com/search?client=firefox-bd\&q=Revista

+de+Educaci\%C3\%B3n+eHumanidades.+Sobre+el+mito+del+amor+rom $\% \mathrm{C} 3 \% \mathrm{~A} 1 \mathrm{ntico}+$ Amores+cinematogr\%C3\%A1 ficosy+educaci\% $\mathrm{C} 3 \%$ B3n $\% 2 \mathrm{C}+\% 2810 \% 29 \% 2 \mathrm{C}+63-78$. 